\title{
Les négociations diplomatiques au Conseil de sécurité
}

\author{
David Ambrosetti (CNRS, ISP)
}

Mots-clés : chapitre VII, sanctions (internationales), lead / leader (diplomatique), veto (droit de), CPI (Cour pénale internationale), régional / régionaux (groupe(s)), consensus / unanimité, comité (de suivi des sanctions / d'experts), mission de paix / mission permanente / mission du Conseil.

\footnotetext{
"In a rational world, one feels, no government in its right mind would ever volunteer to fill an elective Council seat. To do so means that for two years a small state, which presumably has no wish to get into needless quarrels, is liable to have to take a public stand on various awkward questions that are of no direct concern to it, thereby risking the anger of one or more of the great powers and perhaps of other states too."
}

Andrew Boyd, Fifteen Men on a Powder Keg: A History of the U.N. Security Council, p. 3.

Si on en croit Andrew Boyd, cité en exergue, le Conseil de sécurité de l'ONU est un lieu générateur de grands tracas, à tout le moins pour les représentants de «petits États » membres. C'est aussi et surtout une arène multilatérale où se joue une part conséquente de la politique internationale contemporaine. Dans le présent chapitre, nous voulons contribuer à caractériser les pratiques de négociation diplomatiques qui y prennent place. Faire l'inventaire de l'ensemble de ces pratiques serait sans doute illusoire, tant elles inondent en masse cet espace. Pour en explorer quelques caractéristiques, nous repérons dans les lignes qui suivent 
des éléments structurels qui sont susceptibles de donner à ces négociations leurs formes particulières, et exposons certaines de ces pratiques les plus emblématiques à nos yeux.

Le premier élément structurel guidant notre démarche concerne l'objet même de cette instance multilatérale : le maintien de la paix et la sécurité internationales, par le recours à des moyens coercitifs si nécessaire. Un tel mandat, qui en fait un haut lieu de la politique internationale, imprime sa marque dans les négociations diplomatiques qui s'y tiennent, et dans les règles formelles et pratiques autour desquelles celles-ci s'organisent. Négocier au Conseil, c'est avant tout s'accorder sur la définition des menaces et sur les modalités appropriées d'exercice de la coercition. On le rappellera, les «possibles » en la matière s'en sont trouvés grandement modifiés au fil du temps et des débats.

Le deuxième élément qui façonne le cours des négociations réside dans les règles d'accession à la table du Conseil, autrement dit dans la composition de ce dernier. Ces règles déterminent les statuts des membres (entre les permanents et les autres), et plus largement les types de ressources dont ils disposent au cœur de la négociation, qui à leur tour contraignent les pratiques de négociation. Ces ressources ne se limitent pas aux capacités matérielles en soi : il faut par exemple considérer l'inégal accès aux différentes positions prévues par la division sociale du travail multilatéral (telles que le rôle de rédaction des projets de résolution).

Un dernier ensemble d'éléments s'intéresse enfin au "comment» (après le «quoi » et le « qui ») : dans le détail de l'organisation concrète des débats au Conseil (depuis l'inscription à l'ordre du jour jusqu'aux textes votés), nous identifions quatre traits qui nous semblent prégnants, et révélateurs de ce que signifie négocier dans ce cadre institutionnel précis. Ces traits renvoient à la traditionnelle quête du consensus et aux turbulences que celle-ci endure aujourd'hui, à la fréquente désignation de «pilotes» chargés de porter des dossiers spécifiques pour le compte du groupe (le «lead» diplomatique), à l'augmentation de la charge de travail et du niveau de technicité requis de la part des négociateurs qui souhaitent rester influents, et enfin à l'enjeu d'ouverture du Conseil sur l'extérieur, source de contraintes mais également de nouvelles opportunités pour les négociateurs.

Le regard que nous portons sur les négociations au Conseil de sécurité n'est pas neutre : il s'est nourri non seulement de la fréquentation des ouvrages de référence, mais aussi des travaux que nous avons consacrés à cet organe multilatéral, ainsi que de l'observation sur le 
long cours d'un domaine d'action particulier du Conseil, la régulation des conflits armés qui touchent le continent africain ${ }^{1}$.

\section{Négocier au nom de la paix et la sécurité internationales : l'usage de la coercition dans tous les esprits}

En dépit de la lettre de son mandat, le périmètre de compétences du Conseil a varié dans le temps, de même que les types de réponses adoptées, selon les acceptions admises des menaces à « la paix et la sécurité internationales ». Mais c'est bien la prérogative du recours à la coercition pour réguler l'exercice de la violence entre États, voire dans les États, qui semble borner les pratiques de négociations entre États membres. De cette aptitude dérive un ensemble d'enjeux pratiques qui nous semblent particulièrement structurants.

\section{Stabiliser l'ordre international: la variation des menaces et des réponses armées}

Pendant la Seconde Guerre mondiale, les Alliés envisagent pour l'après-victoire militaire une refonte du paysage institutionnel mondial pour la stabilisation de l'ordre international d'aprèsguerre. Le nouveau Conseil de sécurité prend la forme d'un Directoire mondial qui reconnaît, par réalisme politique, le rôle prééminent des cinq grandes puissances victorieuses, et leur octroie un droit de veto. Mais il renoue également avec l'idéal libéral de sécurité collective que le président américain Wilson a incarné au sortir du premier conflit mondial et que la Société des nations a ensuite tenté d'instituer, avant d'être balayée par les puissances « révisionnistes» de l'Axe. L'ONU et son Conseil de sécurité naissent comme une organisation intergouvernementale universelle, permanente, permettant d'associer, directement ou non, tous les États souverains de la planète à la décision y compris dans le domaine ô combien stratégique de la sécurité internationale ${ }^{2}$. Les États-Unis y voient une

\footnotetext{
${ }^{1}$ Cette partie du monde constitue une entrée pertinente pour l'analyse des pratiques quotidiennes du Conseil de sécurité : à la fin des années 2000, $70 \%$ des casques bleus y sont déployés et $60 \%$ des délibérations portent sur ce continent (Adebajo, 2010: 59).

${ }^{2}$ Novosseloff (2003).
} 
« question vitale de politique étrangère » et investissent massivement le travail diplomatique de préparation des discussions et des textes fondateurs ${ }^{3}$.

Les dispositions contenues dans le chapitre VII de la Charte constituent la grande innovation de la Charte de l'ONU puisqu'elles autorisent le recours à des moyens coercitifs pour préserver la paix et la sécurité internationales. Le Conseil entre donc dans la «haute politique » par excellence, où se jouent la guerre et la paix, ou encore le contrôle de la prolifération nucléaire ${ }^{4}$. Mais un mandat ne suffit pas à faire une pratique. En atteste la fréquence du recours au chapitre VII, progressivement tombé en désaffection, avant d'être amplement réinvesti depuis $1990^{5}$.

Qualifier un sujet de «menace à la paix et la sécurité internationales », condition nécessaire à l'inscription de ce sujet à l'ordre du jour, renvoie de fait à la force performative d'un acte de langage. La place laissée à la négociation n'en est que plus grande. La plasticité des termes du mandat pour la définition des menaces ouvre une variation symétrique des types d'actions pacifiques et surtout coercitives que le Conseil est amené à décider. Un rapide inventaire permet de le rappeler. Très rarement pris en considération depuis 1960 (et la mission de l'ONU au Congo), les conflits violents intra-étatiques (et régionaux) constituent une part considérable de l'activité du Conseil depuis la fin de la guerre froide. Cette période récente a également élargi les types d'activités militaires autorisées par le Conseil. Après les coalitions armées autorisées à faire la guerre au nom de l'ONU (Corée en 1950, Irak en 1991, Libye 2011), après l'interposition de soldats de la paix (depuis Suez en 1956) placés sous le commandement politique direct du Secrétaire de l'ONU (dès l'ONUC au Congo) pour « geler» les combats et favoriser le dialogue politique entre belligérants, voire reconstruire l'État, les membres du Conseil se sont prononcés sur l'opportunité de déployer des personnels militaires et civils pour des missions de plus en plus « multidimensionnelles » mêlant moyens coercitifs et moyens pacifiques (avec ou sans le consentement des parties), poursuivant des objectifs de plus en plus variés ${ }^{6}$. A ces agendas politiques, militaires, sécuritaires, administratifs, humanitaires déjà bien chargés s'est ajoutée la prise en compte des activités

\footnotetext{
${ }^{3}$ Novosseloff (2003); Frederking (2007).

${ }^{4}$ Roberts (2008); Sarooshi (1999).

${ }^{5}$ Entre 5 et $10 \%$ des résolutions en moyenne invoquent le chapitre VII avant 1990 . Cette moyenne s'élève à $25 \%$ entre 1990 et 2002, avec 47\% pour l'année 2002 (Wallensteen et Johansson, 2004: 19).

${ }^{6}$ Novosseloff (2003); Bellamy, Williams et Griffin (2004); Howard (2008).
} 
des réseaux criminels et des groupes islamistes radicaux susceptibles de prospérer à l'ombre des « États faibles », ou encore les questions de « sécurité environnementale » ${ }^{7}$.

\section{D'autres instruments de coercition}

Le Conseil dispose également de mesures coercitives alternatives au recours à la force armée. Les sanctions internationales, d'abord, se sont banalisées depuis la fin de la guerre froide, après avoir été expérimentées contre les seules Rhodésie et Afrique du Sud avant 1990. Pour chaque régime créé, le Conseil institue un comité $a d$ hoc de suivi du régime. La présidence de ce comité est confiée par le Conseil, pour un an renouvelable, à un diplomate (souvent un ambassadeur) siégeant au Conseil et ressortissant d'un État membre non permanent, et ce à titre personnel (il ne peut être remplacé par un adjoint). En tant que président du comité, ce diplomate reçoit ses instructions du Conseil et non de sa tutelle. La prise de décision obéit à la règle du consensus ${ }^{8}$.

Cette évolution n'est pas allée sans controverse. Ce fut particulièrement le cas des sanctions contre l'Irak à la fin des années $1990^{9}$. Parce que les régimes de sanction ne prévoyaient alors aucune borne temporelle, les membres du Conseil qui pouvaient souhaiter un réaménagement ou la suspension temporaire ou définitive de ces sanctions pour des motifs humanitaires ou politiques avaient perdu la main: les délégations les plus attachées à la poursuite des sanctions en l'état (États-Unis et Royaume-Uni) étaient par leur droit de veto en mesure de bloquer toute révision de ce régime. Les États membres insatisfaits (menés par la France, la Russie et la Chine) ont obtenu que les régimes de sanction nouvellement créés en $2000^{10}$ le soient sur la base d'un mandat limité dans le temps, permettant à terme échu la reprise des débats sur l'efficacité de ces sanctions et sur l'opportunité de les reconduire en l'état ${ }^{11}$.

\footnotetext{
${ }^{7}$ Sur ce dernier point, $c f$. Marcou (2012).

${ }^{8}$ Van Walsum (2004).

${ }^{9}$ Gordon (2010); Malone (2006).

${ }^{10}$ Dès la résolution 1298 de mai 2000 sur l'Éthiopie et l’Érythrée.

${ }^{11}$ Sur ces débats au sein du groupe de travail sur la réforme des sanctions créé au Conseil en 2000, $c f$. Cortright et Lopez (2004: 175-176). Cf. également Châtaigner (2005) à propos de la résolution 1306 de juillet 2000 sur les sanctions visant les diamants de Sierra Leone.
} 
L'autre nouveauté dans les moyens coercitifs envisagés depuis les années 1990 est le recours à la justice pénale internationale. Sur le modèle des tribunaux ad hoc créés par les Alliés à Nuremberg et à Tokyo après la Seconde Guerre mondiale, le Conseil a, au cours des années 1990, institué deux tribunaux pénaux internationaux, pour l'ex-Yougoslavie (TPIY) puis pour le Rwanda (TPIR), pour punir ceux qui ont commis, ordonné ou autorisé sous leur responsabilité hiérarchique des violences interdites par le droit international humanitaire (crime de guerre, crimes contre l'humanité et génocide). Depuis juillet 2002, le Conseil dispose du droit de saisir la nouvelle Cour pénale internationale (CPI) permanente née à cette date à la Haye pour la répression de ces trois crimes internationaux.

En principe, cet « outil» coercitif échappe au Conseil, une fois saisi. Dans les faits, le propos doit être relativisé pour au moins trois raisons. D'abord, les négociations préalables entre membres du Conseil de sécurité ont toutes les chances d'inclure, par anticipation, l'identité des responsables politiques et militaires les plus exposés au risque d'inculpation. Ensuite, le bureau du procureur dépend largement du soutien des États pour l'exécution de ses décisions. Le Conseil peut ainsi faire pression sur des États afin qu'ils se conforment à telles décisions de la CPI. Dans le même ordre d'idées, le Conseil peut demander à des forces de maintien de la paix sous commandement de l'ONU qu'elles servent de services de renseignements sur le terrain au profit du Procureur de la CPI, voire même de force de police en charge de la traque et la détention provisoire de personnes inculpées, comme l'a montré la FORPRONU en exYougoslavie. Il revient alors au Conseil de convaincre (en lien avec les services du Secrétaire général de l'ONU) les États contributeurs de troupes de mettre en œuvre ce mandat. Il reste enfin une dernière prise dont dispose le Conseil après saisine de la Cour : l'article 16 du statut de Rome de juillet 1998 instituant la CPI prévoit que le Conseil puisse, par le vote d'une résolution, obtenir de celle-ci qu'elle suspende pendant un an (renouvelable) une procédure en cours.

La relation à la CPI produit au moins deux pratiques de négociation intéressantes. Toutes deux concernent en l'espèce la délégation des États-Unis. Tout d'abord, l'administration américaine du président Bush a non seulement refusé de ratifier le statut de Rome, mais elle a également exigé du Conseil une résolution garantissant l'immunité des (rares) personnels américains servant sous mandat de l'ONU vis-à-vis de la CPI (résolution 1422 du 12 juillet 2002). Pour l'obtenir, elle a dû menacer de torpiller, par l'usage de son droit veto, toutes les missions de paix dont le mandat arrivait alors à échéance, sans oublier de promettre des 
représailles diplomatiques à des États membres qui étaient alors candidats à une adhésion à l'OTAN $^{12}$. Depuis l'échec de cette démarche en 2004, la solution promue (y compris sous l'administration Obama) consiste à exiger que toute résolution du Conseil créant ou renouvelant une opération de paix de l'ONU inclue un alinéa disposant explicitement qu'aucun des personnels étatsuniens servant dans ces missions ne pourra être extradé vers la CPI. Seconde pratique intéressante, la position de cette délégation devient plus inconfortable si elle veut au contraire soutenir la saisine de cette Cour. Une solution, utilisée par exemple pour la saisine de la CPI à propos des crimes commis au Darfour (résolution 1593 du 31 mars 2005), consiste à voter en faveur de la résolution à l'exception de l'alinéa demandant la saisine de la CPI, alinéa sur lequel les États-Unis s'abstiennent.

Après l'objet du Conseil, un autre aspect structurant pour les pratiques de négociation réside dans sa composition, dans les règles d'accession à cette enceinte.

\section{Membres permanents, membres élus: plusieurs cercles dans une même enceinte}

Si le formalisme diplomatique qui règne à l'ONU veille à maintenir le principe d'égalité entre États membres, il n'empêche pas les écarts de ressources, ni même de statuts, entre les quinze délégations diplomatiques : cinq doivent leur présence à leur statut de membre permanent, quand les dix autres y siègent seulement pour deux années, au titre de leur élection à l'Assemblée générale des Nations Unies (AGNU) à la majorité qualifiée (des deux tiers).

En ce qui concerne le « $\mathrm{P} 5 »^{13}$, son privilège est double. D’une part, sa présence permanente à la table du Conseil lui vaut une excellente connaissance et une mémoire des précédentes négociations informelles concernant les dossiers. Les autres États membres souffrent d'une présence intermittente (au mieux) au Conseil, ce qui les affaiblit dans les négociations. Ils dépendent dès lors davantage des informations et analyses fournies par le Secrétariat de l'ONU. Cette inégalité les atteint également dans leur capacité de nouer des relations de confiance avec les personnels du Secrétariat précisément, une relation fondamentale dans la

\footnotetext{
12 Ambrosetti (2004).

${ }^{13}$ Le « P5 » constitue un club informel reconnu comme tel depuis 1986.
} 
préparation des décisions du Conseil, qui bénéficie aujourd'hui essentiellement aux membres permanents $^{14}$.

D'autre part, le droit de veto reconnu aux membres permanents (art. 27 de la Charte, qui exclut les questions de procédure, en théorie) accorde à ces derniers un pouvoir considérable. Il constitue une borne limite à la négociation. Cette borne n'est pourtant pas si fréquemment rencontrée. Son utilisation a radicalement décru dès $1990^{15}$. Les Britanniques et les Français en usent avec parcimonie étant donné les jalousies que suscite leur siège permanent dans d'autres chancelleries. Pékin s'est également montré prudent en la matière depuis son entrée au club des permanents en 1971. De façon symbolique, les puissances émergentes les mieux placées pour bénéficier d'un futur élargissement du nombre de sièges permanents, parmi le « G4 » (Allemagne, Brésil, Inde et Japon) mais aussi sur le continent africain (Afrique du Sud et Nigeria), envisageaient en 2005 la possibilité de renoncer au droit de veto pour rallier les membres permanents les plus réticents à cette réforme ${ }^{16}$. Même sans droit de veto, cette présence permanente constitue un bien convoité par ces puissances émergentes. Elle leur garantirait une maîtrise des dossiers, et multiplierait leurs chances de «tenir la plume » dans la rédaction des projets de résolutions importantes, une activité (drafting) largement monopolisée par les permanents ( $c f$. infra). Autant d'éléments pour peser dans les négociations et orienter les choix du Conseil.

En ce qui concerne les États membres non permanents, la question de savoir qui négocie au Conseil est une question... qui se négocie, âprement. Les modalités d'élection méritent ici l'attention car elles influent sur la structure de la négociation, sur la complexité des intérêts en présence. Avant son élection formelle à la majorité qualifiée, par vote à bulletin secret de chaque représentant permanent siégeant à l'AGNU, chaque nouveau membre a dû bénéficier d'une présélection par le Conseil de sécurité. Celui-ci établit une liste de deux à trois candidats pour chaque siège à pourvoir. Ces candidats doivent ensuite être départagés par leur groupe régional d'appartenance, avant le vote formel. Première conséquence, un membre élu

\footnotetext{
${ }^{14}$ Malone (2004: 7).

${ }^{15}$ Ce droit a été utilisé 272 fois entre 1946 et 1989, et 28 fois seulement entre 1990 et 2011, dont 17 fois par les États-Unis (question palestinienne) (Quilès et Novosseloff, 2011).

${ }^{16}$ Cette proposition suscita notamment l'hostilité d'États africains, au nom de la position commune adoptée par l'Union africaine en mars 2005 sur la réforme de la composition du Conseil (le «Consensus d'Ezulwini») (Adebajo, 2010: 58-62). Sur la position chinoise, cf. Panda (2011).
} 
s'est assuré du soutien de ses futurs pairs au Conseil, et surtout il ne souffre pas de l'hostilité ouverte d'un membre permanent, qui pourrait bloquer sa candidature ${ }^{17}$. Seconde conséquence, derrière chaque membre élu réside un des cinq groupes qui réunissent les États de l'ONU sur une base régionale. Chacun de ces groupes a élu un ou plusieurs de ses membres pour siéger au Consei $1^{18}$. Or les États finalement élus portent aussi la voix de leur groupe régional. Pour être élus, ils ont dû engager d'intenses négociations au sein de ce groupe pour emporter son soutien, et ce en élaborant des programmes électoraux qui pourront leur être opposés sur des dossiers spécifiques, une fois élus ${ }^{19}$. Un représentant permanent mexicain pourra ainsi, dans une négociation difficile, se prévaloir du soutien de ses voisins ${ }^{20}$. La pratique est très fréquente à propos des représentants européens, qui s'appuient souvent sur les positions prises par l'Union européenne (UE) ${ }^{21}$, et qui d'ailleurs organisent des rencontres informelles de coordination avant de se rendre au Conseil lorsqu'une négociation intéresse l'UE dans son ensemble ${ }^{22}$. Ces efforts de coordination ne suppriment par les rivalités intereuropéennes pour l'obtention d'un mandat au Conseil.

Un témoignage et une analyse à propos de la campagne « électorale » menée par le Portugal jusqu'à sa victoire confortable en 2010 (pour un siège non permanent en 2011-2012) sont ici riches d'enseignements sur les négociations onusiennes ${ }^{23}$. D'abord sur le degré d'anticipation aujourd'hui nécessaire : c'est en 1999, alors que le Portugal vient de terminer un mandat de membre du Conseil de sécurité (1997-98), que fut lancée cette campagne pour une prochaine mandature. Le créneau de 2011-2012 n'avait été alors convoité par personne. Cette

\footnotetext{
${ }^{17}$ Sur les 193 États membres de l'ONU en 2012, soixante n'y ont jamais siégé, quarante y on siégé seulement une fois. Le Brésil et le Japon y ont, eux, siégé dix fois, l'Argentine, huit, le Canada, l'Inde, l'Italie et le Pakistan, six. $C f$. le site officiel du Conseil de sécurité de l'ONU, http://www.un.org/sc/members.asp/ (consulté le 15 juillet 2012). Sur le Brésil, $c f$. Albaret (2010).

${ }^{18}$ Sur les dix sièges à pourvoir (par moitié chaque année), cinq vont aux États d'Afrique (en général trois) et d'Asie (deux), deux aux États d'Amérique latine et Caraïbes, un aux États d'Europe orientale, et enfin deux au groupe composé des États d'Europe occidentale « et autres États».

${ }^{19}$ Sur la campagne menée par le gouvernement canadien autour de la thématique de la sécurité humaine dans les années 1990, cf. Cathelin (2008).

${ }^{20}$ Nous pensons à l'ambassadeur Zinser et au Groupe de Río dans la négociation décrite dans Ambrosetti (2004).

${ }^{21}$ Précisons que les membres de l'UE se répartissent dans trois groupes régionaux : Europe orientale, Europe occidentale et autre, et... Asie (Chypre) !

${ }^{22}$ Ambrosetti (2004).

${ }^{23}$ Amaral, Martins, et Macieira (2010).
} 
candidature précoce - dix-huit ans avant la date prévue de l'élection - permettait de capitaliser l'appréciation positive portée sur le mandat portugais de 1997-98, mais aussi de bénéficier de la future présidence portugaise de l'Union européenne pour rallier les soutiens des États voisins, tout au laissant le temps aux autres États membres du groupe (Canada, Australie, Nouvelle-Zélande) d'occuper ce siège. Cette tendance à l'anticipation s'est renforcée depuis. Des États ont déjà manifesté leur intérêt pour un mandat en... 2025-2026. Ces campagnes extrêmement longues ne vont pas sans coûts et sans aléas, en particulier face aux alternances politiques qui risquent de faire voler en éclat des ralliements obtenus. Les ressources diplomatiques investies n'en deviennent que plus importantes, augmentant d'autant le coût du ticket d'entrée dans la course. Une fois lancée, la campagne électorale se joue pour beaucoup dans les multiples appartenances des États candidats à des regroupements d'États (régionaux, politiques, thématiques...) et dans la visibilité qu'une diplomatie sait gagner dans ces groupes. Pour mobiliser les alliés autour de leur candidature, les autorités portugaises les ont d'ailleurs impliqués dans la définition des lignes défendues dans cette campagne et dans les actions promotionnelles menées.

Ce statut différencié entre membre permanent et membre élu est crucial, mais il n'est pas le seul clivage significatif. L'identité du négociateur se joue également dans les positions de l'État qu'il représente dans le rapport des forces (de production et de destruction) actuel. On songe au clivage Nord / Sud (pays issus des décolonisations), qui prend forme à l'ONU via les regroupements d'États que sont le G77 et le Mouvement des non alignés (la Chine s'étant traditionnellement présentée comme le champion de ces derniers). La nouveauté des années 2000 et 2010 réside bien dans la reconfiguration des lignes de tension entre Nord et Sud, par le jeu de puissances émergentes de plus en plus autonomes. En résultent par exemple de nouveaux clivages entre émergents et pays les plus pauvres, mais également des confrontations plus dures entre ces émergents profondément réalistes et les États occidentaux « postindustriels » alignés sur des positions plus idéologiques (gouvernance démocratique libérale) et plus interventionnistes (tentés par le « regime-change »).

Nous avons débuté le propos en examinant les enjeux de négociations diplomatiques qui se nichent dans la définition même de l'objet du Conseil, de son mandat et surtout de ses prérogatives dans le recours à la coercition. Nous avons poursuivi en nous interrogeant sur les effets de la composition de cette instance multilatérale et des règles d'accession à un siège de membre sur les pratiques de négociations en présence. Une fois ces éléments structurels bien 
assis, nous pouvons désormais, dans une dernière section, envisager une ultime série d'enjeux de la négociation propres aux processus de prise de décision au Conseil de sécurité.

\section{Au cœur de l'organisation des débats : quatre traits}

Retracer le parcours d'une décision du Conseil (résolutions, déclarations présidentielles, etc.) conduit l'observateur à la rencontre d'un grand nombre de pratiques de négociation, par définition. Nous avons eu l'opportunité de reconstituer dans un précédent texte quelques règles formelles et informelles autour desquelles s'organisent l'inscription à l'ordre du jour et la prise de décision au Conseil, au vu de nos observations de terrain ${ }^{24}$. Nous souhaitons ici souligner quelques traits saillants identifiés dans ces pratiques. Ces traits concernent le poids de l'unanimité dans les débats, le rôle prépondérant reconnu à certains membres (souvent permanents) assurant un rôle moteur dans les débats, le coût accru du ticket d'entrée pour une implication sérieuse dans les débats du Conseil vu la multiplicité et la haute technicité des enjeux, enfin l'enjeu de transparence et d'ouverture des débats, qui renvoie à la façon dont les membres du Conseil intègrent les acteurs non gouvernementaux dans le jeu diplomatique.

\section{En quête de consensus}

La valeur du consensus inonde le travail quotidien du Conseil. Elle s'impose dès les premières négociations visant à en établir l'ordre du jour. Pour élaborer cet ordre du jour durant le mois d'exercice de sa présidence tournante, le président du Conseil doit obtenir l'aval des quatorze autres délégations pour tout nouveau sujet qu'il souhaite inscrire, alors qu'un sujet précédemment ouvert, et suivi à échéances régulières, s'impose à lui. Pour chaque nouveau sujet, donc, les négociations préalables vont bon train afin de surmonter les possibles réticences.

Une fois le Conseil saisi, la quête du consensus demeure un objectif. C'est une condition nécessaire avant toute déclaration présidentielle du Conseil, et elle reste valorisée pour l'adoption des résolutions. Ceci suppose un fonctionnement bien particulier au cœur des

\footnotetext{
${ }^{24}$ Ambrosetti (2009). Cf. également Bailey et Daws (1998).
} 
consultations privées, fait de comptages permanents dans les têtes pour repérer les moments opportuns pour le passage au vote, fait de discrètes pressions collectives avant toute prise de parole avec des répercussions sur les relations futures entre pairs, sans oublier les silences opportunistes destinés à faire monter les enchères, etc. ${ }^{25}$ De fait, les projets de résolution faisant l'objet de votes négatifs ou même d'abstentions ne sont pas si fréquents, mais le sentiment qu'ils sont en augmentation semble assez partagé, au point de susciter certaines impatiences ${ }^{26}$. Cette tendance se fait plus sensible avec les récents développements liés aux crises des régimes autoritaires arabes (en Syrie aujourd'hui) et aux velléités interventionnistes occidentales que les puissances émergentes considèrent devoir contenir ${ }^{27}$.

\section{Le « lead » diplomatique}

Les réticences à l'égard d'un dossier renvoient tantôt à des positions de principe (le respect du principe de non-ingérence dans les affaires intérieures des États, cher au G77), tantôt à des intérêts politiques bien localisés. Un schéma géopolitique classique est ici censé offrir des garanties de succès, lorsque le membre permanent le plus proche de la crise envisagée, en termes de zones d'influence et de capacités d'action, est celui qui porte la demande d'inscription du sujet à l'ordre du jour auprès du président et de ses pairs. Autrement dit, ces derniers doivent être convaincus que ce membre, souvent le mieux informé et le plus influent auprès des acteurs locaux de la crise, ne viendra pas saborder les efforts réalisés, mais qu'il souhaite au contraire cette discussion, et qu'il y consacrera des moyens. Il devra notamment, grâce à ses relais locaux, veiller à garantir le consentement des autorités légales de l'État touché par la crise. Ce faisant, les membres permanents ont pris l'habitude de fonctionner selon la règle du «donnant-donnant»: les exemples abondent en matière de création de nouvelles missions de paix (Rwanda contre Somalie, Sierra Leone contre République centrafricaine, Soudan contre Côte d'Ivoire...).

\footnotetext{
${ }^{25}$ On en trouvera des illustrations dans Ambrosetti (2004). Pour un regard historique, $c f$. Chai (1977). Nous renvoyons enfin, pour discussion, aux règles de négociation identifiées dans la construction de l'unanimité au Conseil de l'UE (Novak, 2010).

${ }^{26}$ A cet égard, entendre des diplomates américains minimiser en privé cet intérêt du consensus (au nom de la règle majoritaire, jugée plus démocratique) ne semble pas totalement accidentel.

${ }^{27} C f$. par exemple sur le Brésil (Albaret, 2010) ou la Chine (Panda, 2011).
} 
Ceci ouvre la voie à une forme particulière de pilotage des négociations. Nous avons mis en lumière dans des travaux antérieurs que les débats s'adossent souvent à un rôle moteur, quasi « entrepreneurial », assumé par une délégation dans la définition d'une ligne de conduite collective, ce qu'on appelle le «lead». Cette délégation travaille à la rédaction des textes (elle « tient la plume ») et à la recherche active de compromis pour faire progresser le dossier et éviter tout enlisement ou toute coalition de blocage ${ }^{28}$. De fait, les trois États membres permanents occidentaux (États-Unis, France, Royaume-Uni) tiennent la plume dans la plupart des résolutions ${ }^{29}$. Mais des membres élus se voient également proposer cette fonction, dès lors qu'ils ne sont pas suspects de partialité trop flagrante pour une partie contre une autre dans les conflits traités ${ }^{30}$. Et c'est sans oublier les formules de groupes d'États bien constitués qui assurent collectivement un rôle diplomatique moteur dans toutes les arènes multilatérales sur des dossiers spécifiques, tels que les «Groupes d'amis » comprenant des États membres actuels et anciens ${ }^{31}$, le E3+3 sur le nucléaire iranien (France, Allemagne, Royaume-Uni, Chine, Russie, États-Unis), etc.

\section{Un ticket d'entrée coûteux en termes de capacités matérielles et techniques}

Dans ces processus informels qui placent certains en position d'influence au détriment d'autres, un facteur pèse de plus en plus lourd : il s'agit de la capacité de chaque délégation de faire face à l'intensification des réunions de travail au Conseil et à la technicisation des débats. A l'époque où Andrew Boyd écrit son célèbre ouvrage sur le Conseil (1971), ce dernier se réunit en moyenne une fois par semaine ${ }^{32}$. En 2002, on a compté 238 réunions (y compris certains dimanches quand l'actualité l'exigeait), pour 64 résolutions adoptées ${ }^{33}$. Entre

\footnotetext{
${ }^{28}$ Ambrosetti (2009, chapitre 5 ; 2012).

${ }^{29}$ C'est ce que confirme Ioannis Mallikourtis, qui fut secrétaire d'ambassade à la Mission permanente de la Grèce à l'ONU quand ce pays siégeait au Conseil (2005-2006), dans une communication au workshop sur « The UN Security Council : A Case Study in Multilateral Diplomacy », organisé en mars 2007 à l'Université de Columbia, School of International and Public Affairs. Selon ce témoin, $80 \%$ des drafts présentés au Conseil au cours de ces deux années avaient été rédigés par ces trois États permanents occidentaux.

${ }^{30}$ Toujours selon Ioannis Mallikourtis.

${ }^{31}$ Whitfield (2007).

${ }^{32}$ Boyd (1971)

${ }^{33}$ Hulton (2004: 239).
} 
2005 et 2006, 158 projets de résolutions auraient été rédigés et présentés au Conseil ${ }^{34}$. Le travail quotidien s'est transformé du fait de l'accroissement considérable des activités du Conseil, en nombre de dossiers inscrits à l'ordre du jour, mais aussi du fait de la diversification des actions qu'il met en place et des méthodes de travail qu'il adopte.

Sur l'aspect proprement quantitatif, cela se traduit par un personnel toujours plus important requis dans les missions permanentes. Mais toutes ne peuvent pas mobiliser plus d'une centaine d'agents, comme le font les États-Unis dans leur représentation permanente à New York $^{35}$. En outre, ces agents doivent mobiliser des compétences toujours plus vastes, et pointues, pour suivre les implications techniques (opérationnelles, juridiques, etc.) des décisions prises par le Conseil, ce qui suppose des savoirs juridiques, économiques, militaires, administratifs, policiers, dans le renseignement, dans l'assistance électorale, dans le suivi des sanctions, dans la réforme de l'État, etc., autant de compétences qui sont puisées dans les autres ministères et administrations publiques des États qui sont suffisamment dotés.

\section{L'exigence d'efficacité et de transparence}

Après le renouveau du Conseil de sécurité en 1991 et les fiascos qui s'en sont suivis dans le domaine du maintien de la paix (Somalie, ex-Yougoslavie, Rwanda, Angola...), les méthodes de travail ont été critiquées et reformulées. Une clef des échecs passés semble résider dans la perte de contrôle des actions autorisées par le Conseil, au profit des coalitions ad hoc ou du Secrétariat de l'ONU, une fois les résolutions votées ${ }^{36}$. Ont donc été recherchés des moyens pour responsabiliser davantage la délégation «leader » qui plaide au Conseil en faveur d'une action collective sur un dossier en relation étroite avec le Secrétariat de l'ONU. Nous exposons une solution multilatérale classique, qui consiste à exiger que le périmètre de la négociation relative à une "grande décision » soit décomposé en séquences ou secteurs, et soumis à conditions. Par exemple, lors du déploiement de la mission de l'ONU en RDC, la MONUC, au tournant des années 2000, des délégations ont accepté de soutenir ce projet à la

\footnotetext{
${ }^{34}$ Selon Ioannis Mallikourtis ( $c f$. note 29).

${ }^{35}$ Dans d'autres représentations permanentes, les personnels se comptent sur les doigts d'une main, et il arrive même qu'ils ne soient pas payés durant des mois (y compris les Représentants permanents).

${ }^{36}$ Berman (2004).
} 
condition que ce déploiement soit découpé en plusieurs phases, où le passage d'une phase à l'autre serait soumis au respect de conditions, acté par le vote d'une nouvelle résolution.

Le Conseil doit se mettre davantage à l'écoute des acteurs qui comptent sur un dossier (acteurs politico-militaires du conflit, États voisins, acteurs de la «société civile » locale et internationale, témoins, États contributeurs de troupes, filières économiques affectées par des sanctions, etc.). Voilà les multiples regards que l'«avocat » d'un dossier au Conseil et son président doivent être en mesure d'apporter à leurs pairs pour garantir la mobilisation durable de ces derniers. Sont ainsi organisés des missions du Conseil sur le terrain pour collecter des données ou négocier directement avec les acteurs locaux ${ }^{37}$, des «workshops » en séances publiques avec les contributeurs de troupes ou sur des thèmes transversaux, des invitations de représentants d'États voisins et d'acteurs opérationnels (du Secrétariat et d'agences de l'ONU) à participer à des séances de consultation privées (sans droit de vote), et même des séances informelles hors l'enceinte du Conseil dédiées à auditionner des acteurs non officiels, témoins, représentants d'ONG, etc. (les fameuses séances initiées en mars 1992 par l'ambassadeur vénézuélien Diego Arria, devenues éponymes) ${ }^{38}$.

Le recours aux comités d'experts est devenu un levier important dans les stratégies argumentatives des négociateurs. Ces comités permettent de verser aux débats des éléments «objectifs» sur les comportements de gouvernements, de groupes armés ou de sociétés commerciales, en particulier pour le suivi des sanctions internationales. La publication de rapports d'enquête peut également marquer des temps forts dans les stratégies de négociation, puisqu'elle peut générer quelques répercussions dans les organes de presse internationaux, et en particulier dans le New York Times, si influent dans les couloirs de l'ONU que David Malone le qualifie de « council's 'home' broadsheet $»^{39}$. La composition de ces comités n'en

\footnotetext{
${ }^{37}$ Trente-sept missions de ce genre, qui conduisent les représentants permanents à s'envoler sur les théâtres d'intervention du Conseil, ont été organisées de 1946 à 2003, dont la moitié à partir de 1993 et treize à partir de 2000 (Hulton, 2004: 240). Le site officiel du Conseil de sécurité liste 39 rapports de missions du Conseil de sécurité depuis octobre 1992 à janvier 2011. http://www.un.org/Docs/sc/missionreports.html (consulté le 15 juillet 2012).

${ }^{38}$ Soixante-dix réunions « Arria » ont été organisées de 1993 à 2000, mais seulement trois fois pour écouter des ONG. En 2001-2002, ce sont quatorze de ces réunions qui se sont tenues avec des représentants d'ONG (Hulton, 2004: 243-244).

${ }^{39}$ Malone (2004: 13).
} 
devient que plus stratégique. Elle met en jeu la qualité des relations existant entre les délégations membres du Conseil et les équipes du Secrétariat de l'ONU, qui composeront ces comités.

Dans ces différentes tendances, on le voit, l'enjeu de la publicité, de l'ouverture au regard extérieur, semble déterminant. Après des années 1990 plus avares en séances publiques au profit de consultations privées, la fréquence des séances publiques s'est accrue. Le Conseil publie désormais son programme mensuel. Les verbatim des séances publiques sont accessibles en ligne. L'association plus étroite d'experts et d'ONG au travail quotidien des délégations y participe ${ }^{40}$. D'autres diront que le jeu diplomatique absorbe désormais ces nouveaux relais comme autant de leviers pour agir sur les partenaires-rivaux, pour peser sur l'information dont ils disposent, et pour les placer devant des audiences plus larges sources de pressions nouvelles, par «sociétés civiles » interposées ${ }^{41}$. Un bon négociateur sait donc qu'il doit également compter sur ses bonnes relations avec des porte-parole légitimes de la société civile et de médias influents intéressés par les dossiers qu'il traite, et ce pour faire indirectement progresser ses vues à la table du Conseil.

\section{Conclusion}

Divers enjeux de la négociation ont émergé de cette rapide excursion aux côtés des diplomates siégeant au Conseil de sécurité de l'ONU : la qualification des situations et des réponses légitimes, les statuts différenciés entre négociateurs, les appartenances diplomatiques plurielles (régionales, économiques, politiques, thématiques) qui contraignent le travail de représentation, le coût d'entrée requis pour accéder à certains niveaux d'influence, le poids du contrôle de l'information et de l'accès à une mémoire institutionnelle, le savant dosage de l'explicite et de l'implicite au cœur des débats, l'articulation des relais extérieurs à l'arène diplomatique, parmi d'autres points.

$\mathrm{Au}$ terme de ce travail, on voit que le Conseil de sécurité ne constitue pas un terrain diplomatique entièrement à part, mais il n'en reste pas moins marqué par les particularités

\footnotetext{
${ }^{40}$ C'est ce que veut retenir Hulton (2004).

${ }^{41}$ Sharp (2009: 166-167).
} 
structurelles des dossiers qu'on y traite et des acteurs qui y siègent. Les négociateurs ont tout de même connu des mutations de taille dans leur cadre de travail depuis vingt ans, qui ont notamment modifié leur relation à la parole et l'image publiques, ainsi qu'au savoir expert, et qui les ont surtout exposés à une charge de travail bureaucratique sans précédent, parallèlement à la multiplication des activités destinées à accroître la visibilité du Conseil et de ses actions. Il est encore difficile de dire si l'efficacité fondamentale de ces dernières s'en trouve améliorée, d'un point de vue qualitatif.

Il n'était pas dans notre intention de proposer une qualification univoque des pratiques de négociation en comparaison d'autres arènes diplomatiques, ou au regard d'un « art de bien négocier» en général. Le présent texte est davantage conçu comme une invitation à l'exploration, à la curiosité sociologique à l'égard de pratiques de négociation pour le moins « consistantes» en termes empiriques, et aux implications politiques et humaines parfois considérables, pour le meilleur et pour le pire.

\section{Bibliographie}

Adebajo (Adekeye), The Curse of Berlin. Africa after the Cold War, Londres, Hurts \& Co., 2010.

Albaret (Mélanie), Le Brésil et le Mexique dans le multilatéralisme onusien depuis 1945 : les dynamiques circulaires de l'engagement multilatéral, Thèse de doctorat en science politique, IEP de Paris, 2010.

Amaral (José), MARTins (Sara), MacieIRA (Rui), «All for one, as long as there is not one for Europe », Portuguese Journal of International Affairs, 4, automne-hiver 2010, p. 3139.

AmbrosetTi (David), Normes et rivalités diplomatiques à l'ONU. Le Conseil de sécurité en audience, Bruxelles, P.I.E. Peter Lang, 2009. , «The Diplomatic Lead in the United Nations Security Council and Local Actors' Violence: The Changing Terms of a Social Position », African Security, 5 (2), juin 2012, p. 63-87. 
, "'Décide de demeurer saisi de la question'. La mobilisation du Conseil de sécurité de l'ONU face aux crises », Cultures \& Conflits, 75, hiver 2009, p. 99-122.

, «S'opposer aux États-Unis au Conseil de sécurité : l'argumentation contre la puissance dans les négociations multilatérales », Études internationales, 35 (3), septembre 2004, p. 469-494.

BAILEY (Sydney D.), DAWs (Sam), The Procedure of the UN Security Council, Oxford, Clarendon Press, 1998.

BARNETT (Michael M.), «UN Security Council, Indifference, and Genocide in Rwanda », Cultural Anthropology, 12 (4), 1997, p. 551-578.

Bellamy (Alex), Williams (Paul), GRIFFIN (Stuart), Understanding Peacekeeping, Cambridge, Polity, 2004.

Berman (Frank), « The Authorization Model», in David M. Malone (dir.), The UN Security Council: From the Cold War to the $21^{\text {st }}$ Century, International Peace Academy, Boulder, Lynne Rienner, 2004, p. 153-165.

Boyd (Andrew), Fifteen Men on a Powder Keg: A History of the U.N. Security Council, Londres, Methuen \& Co., 1971.

CATHELIN (Mélanie), Le rôle international d'un État: construction, institutionnalisation et changement. Le cas de la politique canadienne de maintien de la paix en Afrique, Thèse de doctorat en science politique, IEP de Bordeaux, 2008.

CHAI (Feng Yang), «Consultation and consensus in the Security Council», in K. Venkata Raman (dir.), Dispute Settlement through the United Nations, Dobbs Ferry, Oceana Publications, 1977, p. 517-537.

ChÂTAIgNER (Jean-Marc), L'ONU dans la crise en Sierra Leone. Les méandres d'une négociation, Paris, Karthala, 2005.

Cortright (David), LOPEZ (George A.), «Reforming Sanctions », in David M. Malone (dir.), The UN Security Council: From the Cold War to the $21^{\text {st }}$ Century, International Peace Academy, Boulder, Lynne Rienner, 2004, p. 167-179.

Frederking (Brian), The United States and the Security Council: Collective Security since the Cold War, Londres, Routlegde, 2007.

Gordon (Joy), Invisible War: the United States and the Iraq Sanctions, Cambridge, Harvard University Press, 2010.

Howard (Lise Morjé), UN Peacekeeping in Civil Wars, Cambridge, Cambridge University Press, 2008. 
Hulton (Susan C.), «Working Methods and Procedures », in David M. Malone (dir.), The UN Security Council: From the Cold War to the $21^{\text {st }}$ Century, International Peace Academy, Boulder, Lynne Rienner, 2004, p. 237-251.

Quilès (Paul), Novosseloff (Alexandra), «Droit de veto au Conseil de sécurité : parlonsen !», Le Monde, 9 décembre 2011.

Malone (David M.), The International Struggle over Iraq: Politics in the UN Security Council 1980-2005, Oxford, Oxford University Press, 2006.

Malone (David M.) (dir.), The UN Security Council: From the Cold War to the $21^{\text {st }}$ Century, International Peace Academy, Boulder, Lynne Rienner, 2004.

MARCOU (Pauline), La sécurité environnementale : un enjeu pour le Conseil de sécurité?, mémoire de Master en Carrières européennes et internationales, Sciences Po Lille, juin 2012.

NovaK (Stéphanie), « Decision Rules, Social Norms and the Expression of Disagreement: the Case of Qualified-Majority Voting in the Council of the European Union », Social Science Information, 49 (1), mars 2010, p. 83-97.

Novosseloff (Alexandra), Le Conseil de sécurité des Nations Unies et la maîtrise de la force armée. Dialectique du politique et du militaire en matière de paix et de sécurité internationales, Bruxelles, Bruylant, 2003.

PANDA (Jagannath P.), «Beijing's Perspective on UN Security Council Reform: Identity, Activism and Strategy », Portuguese Journal of International Affairs, 5, printemps / été 2011, p. 24-36.

Roberts (Adam), Selective Security: War and the UN Security Council since 1945, New York, Routledge, 2008.

SARoOSHI (Danesh), The United Nations and the Development of Collective Security: The Delegation by the UN Security Council of its Chapter VII Powers, Oxford, Clarendon Press, 1999.

SHARP (Paul), Diplomatic Theory of International Relations, Cambridge, Cambridge University Press, 2009.

Van Walsum (Peter), « The Iraq Sanctions Committee », in David M. Malone (dir.), The UN Security Council: From the Cold War to the $21^{\text {st }}$ Century, International Peace Academy, Boulder, Lynne Rienner, 2004, p. 181-193.

Wallensteen (Peter), Johansson (Patrik), « Security Council Decisions in Perspective », in David M. Malone (dir.), The UN Security Council: From the Cold War to the $21^{\text {st }}$ Century, International Peace Academy, Boulder, Lynne Rienner, 2004, p. 17-33. 
WhitfiEld (Teresa), Friends Indeed? The United Nations, Groups of Friends, and the Resolution of Conflict, Washington, United States Institute of Peace, 2007. 